cells (RBC) after pre-analytical processing. RBC which lack inosine-monophosphate dehydrogenase, critical for bioconversion of thiopurines, do not reflect thiopurine metabolism in peripheral mononuclear cells (PMC)) as exemplified by the poor concordance between metabolite levels and clinical response. To address this problem we have developed a 'direct method' of measuring thiopurine metabolites in both RBC and PMC.

Methods PMCs and RBCs were isolated from blood samples of thiopurine (low dose aza/allopurinol (LDAA), TG or MP) treated patients. They were separated by Lymphoprep, sonicated, centrifuged and $50 \mathrm{uL}$ of supernatant injected for chromatographical separation of the metabolites and analysed on a API4000 triple quadrupole LC-MS/MS. Standard curves and controls validated and metabolite levels reported as $\mathrm{pM}$ of metabolites/mg of protein.

Results Concentrations of metabolites in both RBC and PMCs were determined from standard curves $\left(\begin{array}{lll}7.8 & -500 & \mathrm{nM}\end{array}\right)$ and expressed relative to protein concentration. Comparison between these and results from commercially available RBC metabolite levels are shown below. Sum of methylated metabolites from the LCMS/MS includes methylated thioguanine nucleotides. Undetected metabolites listed as ND

Conclusion The metabolite profiles between patients on FDA, LDAA and TG are very different indicating that these treatments have distinct metabolic pathways. The direct and commercial methods are also different in metabolic profiles raising the suspicion that the commercial method is not an accurate reflection of true metabolic profiles in RBCs. The clinical implication from these data is that the choice of drug protocol (LDAA in "high methylators") is not based on reliable methods. To confirm this, these data and other markers of response and efficacy are being collected prospectively to facilitate a more informed and deeper understanding of how and why FDA, LDAA and TG treated patients respond to these different drug protocols, with an ultimate goal of individualisation of therapy and improvement of the use of these cheap and established drugs.

Disclosure of Interest None Declared.

\section{PWE-100 INCREASING WEIGHT AND BODY MASS INDEX ADVERSELY AFFECT THIOGUANINE NUCLEOTIDE LEVELS IN INFLAMMATORY BOWEL DISEASE}

${ }^{1} S$ Subramanian, ${ }^{1} S$ Poon*, ${ }^{1} A$ Kneebone, ${ }^{2} R$ Asher, ${ }^{2} R$ Jackson, ${ }^{1} B$ Gregg, ${ }^{1} P$ Collins, ${ }^{1} \mathrm{C}$ Probert, 'M Dibb. 'Gastroenterology, Royal Liverpool University Hospital, Liverpool, UK; ${ }^{2}$ Statistics, University of Liverpool, Liverpool, UK

\subsection{6/gutjnl-2014-307263.360}

Introduction Inflammatory bowel disease (IBD) often requires long term immunosuppressive therapy with thiopurines such as azathioprine (AZA) or mercaptopurine (MP) and anti-tumour necrosis factor (TNF) agents. Despite the variable response to thiopurines and anti-TNF agents, few predictive factors of response have been identified. A lower body mass index (BMI) has been associated with a better outcome for azathioprine therapy, infliximab and adalimumab. Obese IBD patients are more likely to have active disease or be hospitalised. This study examined the association between weight and thiopurine therapy by examining 6-thioguanine nucleotide (6-TGN) levels.

Methods We conducted a retrospective analysis of patients who were treated at the Royal Liverpool University Hospital with a thiopurine. The dose of thiopurines was adjusted as tolerated to a maximum of $2.5 \mathrm{mg} / \mathrm{kg}$ for AZA and $1.5 \mathrm{mg} / \mathrm{kg}$ for MP. Eligible patients had a 6-TGN measurement with their height and weight recorded at the same time. Associations between 6-TGN, BMI, weight, patient demographics and biochemical indices were estimated using a multivariable linear regression model. Body fat index was calculated as described previously. All tests were declared statistically significant if $\mathrm{p}<0.05$.

Results 106 patients (48 male, 58 female) were included and contributed 133 measurements. 55\% had Crohn's disease and $45 \%$ had ulcerative colitis. $91 \%$ were on AZA and $9 \%$ were on MP. After adjustment, a one kilogram increase in weight was associated with a 1.62 unit decrease in 6-TGN levels $(95 \% \mathrm{CI}$ : 0.40 to $2.82, \mathrm{p}=0.0094)$. Body fat index correlated strongly with weight for both males and females $(0.8345$ and 0.8860 respectively) and a significant difference was found between BFI for each sex $(p<0.001)$ with females, on average, having a higher BFI. Weight, BMI and BFI differed significantly across sub-therapeutic, therapeutic and supra-therapeutic 6-TGN groups (Table 1).

Conclusion 6-TGN levels decreased significantly with increasing weight and BMI despite a similar weight based dosing. This may explain the previously noted adverse outcomes in obese IBD subjects and underscores the importance of thiopurine metabolite testing

Disclosure of Interest S. Subramanian Speaker bureau with: Speaker honoraria from Warner Chilcott, Shire, MSD, Abbvie and Almirall, Conflict with: Conference support from Abbvie, MSD and Warner Chillcott, S. Poon: None Declared, A. Kneebone: None Declared, R. Asher: None Declared, R. Jackson: None Declared, B. Gregg: None Declared, P. Collins: None Declared, C. Probert: None Declared, M. Dibb: None Declared.

\section{PWE-101 IMAGING THE SMALL BOWEL IN ULCERATIVE COLITIS-RELEVANT OR STILL "UNCLASSIFIED"?}

S Singh*, D Aggarwal, JK Limdi. Gastroenterology, Pennine Acute Hospitals NHS Trust, Manchester, UK

\subsection{6/gutjnl-2014-307263.361}

Introduction It is widely accepted that Ulcerative Colitis (UC) is a mucosal inflammatory disease confined to the colon. Bolder

\begin{tabular}{|c|c|c|c|c|}
\hline \multicolumn{5}{|c|}{ 6TGN Level } \\
\hline & Sub $(<230 \mathrm{pg} / \mathrm{ml})$ & Therapeutic (230-450 pg/ml) & Supra $(>250 \mathrm{pg} / \mathrm{ml})$ & \\
\hline & $\mathrm{n}=63$ & $\mathrm{n}=48$ & $\mathrm{n}=22$ & $p$ \\
\hline Dose per kg: & $1.71(0.65)$ & $1.62(0.66)$ & $1.84(0.70)$ & 0.4641 \\
\hline Weight & $79.3(26)$ & $67.5(14)$ & $62.2(14)$ & 0.0008 \\
\hline Body Mass Index (BMI) & $27.4(9)$ & $23.4(4)$ & $22.6(5)$ & 0.0024 \\
\hline Body Fat Index (BFI) & $31.1(12)$ & $25.6(9)$ & $25.6(11)$ & 0.0199 \\
\hline
\end{tabular}




\begin{tabular}{ll}
$\begin{array}{l}\text { Abstract PWE-101 Table } 1 \\
\text { ulcerative colitis }(\mathrm{n}=47)\end{array}$ & Context of small bowel imaging in \\
\hline Context & $7(15 \%)$ \\
\hline At the time of disease diagnosis (1st episode; equivocal histology) & $17(36 \%)$ \\
Prior to therapy escalation & $9(19 \%)$ \\
Gastrointestinal symptoms atypical for UC & $7(15 \%)$ \\
Abdominal pain (post-colectomy) & $7(15 \%)$ \\
\hline
\end{tabular}

disease definitions coupled with novel and evolving imaging paradigms have led to more sophisticated small bowel imaging in recent years. Despite this, studies on the relevance and yield of small bowel pathology in UC patients are limited.

Methods We conducted a retrospective review of consecutive UC patients seen at our institution between May-October 2013. Clinical data for demographics, disease characteristics, small bowel investigation, endoscopy, treatment and clinical outcomes were obtained from electronic patient records.

Results We analysed 321 patients with biopsy confirmed UC [61\% male, mean age $53 \mathrm{yrs}$, range 18-91 yrs]. Mean age of diagnosis was 43 yrs and mean disease duration was 10 yrs. Montreal classification was E1 (14\%), E2 (49\%) and E3 (37\%) respectively.

Forty-seven patients had small bowel investigations: MR enterography in $81 \%$, CT enterography in $15 \%$ and barium follow-through in $4 \%$. Disease severity at the time of small bowel imaging was mild to moderate in $68 \%$ and $32 \%$ had severe disease. Thirty-two percent of patients were on thiopurines, $19 \%$ on bimodal immunosuppression (infliximab + thiopurine) and $49 \%$ were on aminosalicylates. Seven patients had undergone colectomy.

Small bowel imaging was reported normal in $46 / 47$ and one patient had distal and terminal ileal disease. Indications for small bowel imaging are shown in Table 1 . Subsequent to small bowel imaging, 40\% (19/47) had no change in therapy, 43\% were escalated to immunosuppressive therapy and $11 \%$ underwent surgery for UC. In 3 patients, the diagnosis was changed from UC to Crohn's disease ( 2 with perianal Crohn's; 1 with small bowel Crohn's based on MRE) all subsequently treated with Anti-TNF therapy.

Conclusion The yield of small bowel pathology in our cohort was low, supporting current European Crohn's and Colitis Organisation (ECCO) recommendations. ${ }^{1}$ Small bowel imaging in UC meanwhile should be considered in the well-selected patient and driven by the clinical question or diagnostic uncertainty.

\section{REFERENCE}

1 Dignass A, Eliakim R, Magro $\mathrm{F}$ et al. Second European evidence-based consensus on the diagnosis and management of ulcerative colitis part 1: definitions and diagnosis. I Crohns Colitis. 2012 Dec;6(10):965-90

Disclosure of Interest None Declared.

\section{PWE-102 INFECTIVE DIARRHOEA COMPLICATING IBD VS NON-IBD PATIENTS}

'S Mumtaz*, 'S Kelly, 'UA Ahmad, 'S Everett. 'Gastroenterology, Leeds Teaching Hospitals, Leeds, UK; ${ }^{2}$ Acute Medicine, Doncaster Royal Infirmary, Doncaster, UK

\subsection{6/gutjnl-2014-307263.362}

Introduction Clinical features associated with infective diarrhoea and flare up of Inflammatory Bowel Disease (IBD) are very similar and presents a challenge to gastroenterologists to distinguish the two especially at the initial presentation.

Methods This was a retrospective cohort study for patients admitted to the gastroenterology department of a single tertiary care teaching hospital with a culture positive episode of bacterial infective diarrhoea, excluding Clostridium difficile. Data was collected from clinical notes of patients presenting over an 8 year period (2004-2012). We made comparisons between the cohorts of patients with and without IBD.

Results 103 patients were included in the study; 13 had preexisting IBD (7 UC, 6 Crohn's), mean age [52 (17 - 82) IBD, 41 $(18$ - 103) non- IBD]. Organisms cultured; IBD [campylobacter $84 \%$ (11), salmonella 16\% (2)], non-IBD [campylobacter 81\% (73), salmonella $12 \%$ (11), ecoli 4\% (4), shigella 2\% (2)]. Median duration of symptoms was similar in both groups [4 days (IQR 2) IBD, 4 (IQR 6) non-IBD]. Patient gave history of (IBD vs non IBD); abdominal pain [77 vs. $90 \%$ OR $0.37(95 \%$ CI: $0.08-1.6)$ ], per rectal bleeding [46 vs. $52 \%$ OR $0.78(95 \%$ CI: $0.24-2.5)]$, vomiting $[38 \%$ vs. $43 \%$ OR 0.81 (95\% CI: 0.24-2.71)]. Blood tests showed (IBD vs non IBD); platelet count [median 282 (IQR 130) vs. 237 (IQR 90)], CRP [56 (IQR 81 ) vs. 82 (IQR 102)], haemoglobin concentration [median 12.1 (IQR 2.4) vs. 14.3 (IQR 2.6)] and white cell count [median 8 (IQR 4) vs. 7 (IQR 3)]. A much higher proportion of patients in IBD group received steroids [77 vs 16\% OR 16.6 (95\% CI: 4.061)]. Patients in both group had comparable duration of hospital stay [median 5 (IQR 4) vs. 4 (IQR 3)].

Conclusion Infective diarrhoea presents similarly in patients who have a background of IBD to those that don't but is more likely to be treated with steroids. A short history with abdominal pain and vomiting suggest an infective cause should be considered even if there is a established diagnosis of IBD.

Disclosure of Interest None Declared.

\section{PWE-103 TERMINAL ILEITIS AT ENDOSCOPY IN CLINICAL PRACTICE: IS IT ALWAYS DUE TO CROHN'S DISEASE?}

${ }^{1} \mathrm{~S}$ Zafar*, ' $\mathrm{A}$ Trevatt, ${ }^{2} \mathrm{~A}$ Joshi, ${ }^{1} \mathrm{~K}$ Besherdas. 'Gastroenterology, Chase Farm Hospital, Enfield, Middlesex, London, UK; ${ }^{2}$ Histopathology, Chase Farm Hospital, Enfield, Middlesex, London, UK

\subsection{6/gutjnl-2014-307263.363}

Introduction Terminal ileitis (TI) when identified at endoscopy is generally assumed to be secondary to Crohn's disease (CD). However, TI may be due to other causes including infection, malignancy, radiation, vasculitis and autoimmune disease. In the presence of histological findings such as granulomas, cryptitis, crypt abscesses, fissuring and ulceration, a diagnosis of $\mathrm{CD}$ can be inferred. In the absence of such histological correlation, a diagnosis of $\mathrm{CD}$ may be reinforced by finding inflammation on colonoscopy or small bowel studies in other parts of the gut, suggestive of skip lesions. We hypothesise that patients diagnosed with TI on endoscopy do not all have CD, but may be inaccurately labelled as such.

Methods Single-centre retrospective analysis of 56 patients diagnosed with TI on endoscopy at a district general hospital in London between 2007-2013. Data obtained from endoscopic reporting tool and patient records were correlated with histology and imaging. The number of patients with the finding of ileitis on endoscopy subsequently diagnosed with $\mathrm{CD}$ was studied and the basis of this diagnosis was evaluated.

Results $68 \%$ of patients (38/56) with TI on endoscopy had histological confirmation of ileitis: 11 of whom had characteristic 